\title{
El proceso de toma de decisiones y la eficacia organizativa en empresas privadas del norte de Chile
}

\author{
Decision-making process and organizational performance \\ in private companies in northern Chile
}

\author{
Emilio Rodríguez-Ponce ${ }^{1} \quad$ Liliana Pedraja-Rejas $^{2} \quad$ Carmen Araneda-Guirriman $^{3}$ \\ Recibido 4 de abril de 2013, aceptado 3 de septiembre de 2013 \\ Received: April 4, $2013 \quad$ Accepted: September 3, 2013
}

\begin{abstract}
RESUMEN
El objetivo de esta investigación consiste en describir la relación entre el proceso de toma de decisiones y la eficacia organizativa de las empresas privadas del norte de Chile. Para este efecto, se trabajó con una metodología cuantitativa, donde se aplicó un cuestionario a una muestra de 96 altos directivos de instituciones privadas del extremo norte de Chile, correspondiente a una tasa de respuesta del $22,2 \%$. El muestreo usado fue intencionado. Se llevaron a cabo 3 análisis de regresión lineal, cuyos resultados muestran que la eficacia organizativa es explicada en 59,9\% por la calidad de las decisiones estratégicas $(\mathrm{p}<0,01)$, la calidad de las decisiones estratégicas se explica en $61,5 \%$ por el grado de racionalidad del proceso de toma de decisiones estratégicas $(\mathrm{p}<0,01)$, y el conflicto cognitivo y la flexibilidad cognitiva explican el 76,4\% de la racionalidad del proceso de toma de decisiones $(\mathrm{p}<0,01)$. Por tanto se debe plantear que para lograr mayores niveles de éxito en la toma de decisiones estratégicas y subsecuentemente mayor eficacia organizativa, los equipos de alta dirección deben favorecer la racionalidad de las decisiones estratégicas, buscando y analizando información para la generación de alternativas.
\end{abstract}

Palabras clave: Calidad de decisiones estratégicas, eficacia organizativa, racionalidad, flexibilidad, conflicto cognitivo, proceso de toma de decisiones estratégicas.

\begin{abstract}
The aim of this research is to describe the relationship between decision-making process and organizational performance of private companies in northern Chile. With this purpose a quantitative methodology was applied to a questionnaire addessed to a sample of 96 top management executives of private institutions of northern Chile, corresponding to a response rate of $22.2 \%$. Intentional sampling technique was used. Three linear regression analysis were performed and their results show that organizational performance is explained by $59.9 \%$ for the quality of strategic decisions $(p<0.01)$, the quality of strategic decisions is explained by $61.5 \%$ for the degree the degree of rationality of the process of strategic decision-making $(p<0.01)$ and the cognitive conflict and cognitive flexibility explained $76.4 \%$ of the rationality of the decision-making process $(p<0,01)$. Therefore, it is concluded that the decision-making process includes a number of elements that affect the quality of strategic decisions, the latter being the one related directly and positively to organizational performance of institutions.
\end{abstract}

Keywords: Quality of strategic decisions, organizational performance, rationality, flexibility, cognitive conflict, strategic decision-making process.

\footnotetext{
1 Instituto de Alta Investigación. Universidad de Tarapacá. Casilla 7-D. Arica, Chile. E-mail: erodrigu@ uta.cl

2 Escuela Universitaria de Ingeniería Industrial, Informática y de Sistemas. Universidad de Tarapacá. Casilla 7-D. Arica, Chile. E-mail: lpedraja@uta.cl

3 Centro de Ingeniería aplicada a la Educación. Universidad de Tarapacá. Casilla 7-D. Arica, Chile. E-mail: caraneda@uta.cl
} 


\section{INTRODUCCIÓN}

La ingeniería industrial es de la rama de las ciencias de la ingeniería que se orienta al estudio del desarrollo, mejora, implantación y evaluación de sistemas integrados de personas, riqueza, conocimientos, información, equipamiento, energía, materiales y procesos. En este contexto, la dirección estratégica emerge como disciplina que estudia el proceso de decisiones estratégicas en organizaciones. En dicho proceso los altos directivos de las organizaciones escogen los mercados, eligen la posición competitiva y generan las competencias esenciales. Por consiguiente, las decisiones estratégicas se configuran como elecciones cuyo impacto es de largo alcance. Estas elecciones comprenden el empleo de recursos significativos de las empresas e implican la participación de varios niveles y funciones al interior de las organizaciones [27-28], ya que puede repercutir y afectar su funcionamiento y sus resultados.

En este contexto, el proceso de toma de decisiones considera las alternativas de cursos de acción, el análisis a fondo de todas las opciones estratégicas, y el uso de diversos criterios [37]. Esto con el fin de tomar la mejor decisión que conduzca a los resultados esperados por el equipo encargado de la toma de decisiones.

La relevancia de este proceso radica en que se ha comprobado empíricamente que el $50 \%$ de las decisiones fracasan, lo que sugiere que a pesar de su importancia, adoptar decisiones de calidad no resulta en absoluto un proceso trivial [23-24]. En este sentido, la evidencia empírica también muestra que la calidad promedio de las decisiones adoptadas por diferentes organizaciones promedian una calificación solo regular y distante estadísticamente de niveles óptimos o deseables [32].

A este respecto Nutt [22] considera que la toma de decisiones ha sido tratada en la investigación como estructura o proceso, pero rara vez como ambas. Más aún, Nutt señala que existe una renuencia en tratar la toma decisiones con procesos empíricos, y por ende mucha investigación al respecto ha sido abordada por medio de generalizaciones sobre los procesos, y no por sus pasos de acción. Por consiguiente el autor sugiere que se debe resaltar la importancia del proceso en sí mismo.
Este planteamiento es de suma importancia, ya que con el análisis del tipo de decisión en conjunto y con los estudios de procesos de toma de decisión, los investigadores eventualmente podrían ser capaces de recomendar procesos apropiados o eficientes para cierto tipo de decisiones [10].

En efecto, conocer y comprender los elementos que inciden en el diseño de las decisiones estratégicas es una labor crucial, cuyo resultado puede conllevar a develar las prácticas que producen mejores alternativas de éxito estratégico en las empresas $[14,2]$. Existiendo, por ende, organizaciones que son más exitosas que otras.

Por lo tanto, las decisiones estratégicas son una herramienta crucial en las organizaciones debido a que ellas manifiestan expresamente elecciones a largo plazo que involucran una parte importante de los recursos de la compañía y los esfuerzos de un importante número de personas y funciones.

En este sentido, el objetivo de esta investigación es describir la relación del proceso de decisiones estratégicas y la eficacia organizativa de las empresas privadas en Chile. Para este efecto se aplicó un cuestionario a una población de 320 altos directivos de instituciones privadas de una ciudad del extremo norte de Chile.

Más específicamente, este trabajo tiene como propósito (1) describir la relación entre la calidad de la decisión y el logro de la eficacia organizativa; (2) proporcionar evidencia empírica en un área disciplinaria de exigencia creciente para las empresas; (3) generar recomendaciones para optimizar las posibilidades de éxito en la dirección de las organizaciones.

Por lo tanto, la cuestión a descubrir consiste en identificar de qué manera la calidad de las decisiones se correlacionan con la eficacia organizativa, es decir, en qué medida el fracaso o éxito de las decisiones estratégicas impacta sobre las organizaciones.

\section{MARCO TEÓRICO E HIPÓTESIS}

En la actualidad, las decisiones se configuran como un elemento constitutivo de ventaja competitiva en la sociedad del conocimiento. 
Esto debido a que el pronóstico de un proyecto exitoso y el enfoque de una decisión efectiva pueden, por tanto, facilitar la toma de decisión respecto de la implementación de la gestión del conocimiento [4]. Por esta razón, se puede establecer que la creación de valor estratégico en las organizaciones, se produce en los procesos de toma de decisiones estratégicas [13].

Resulta fundamental entonces analizar cada uno de los aspectos involucrados en el proceso de toma de decisiones y que finalmente tiene un impacto sobre la eficacia de las organizaciones.

Eficacia organizativa y calidad de las decisiones La eficacia organizativa puede ser entendida como el grado por el cual una organización logra realizar sus objetivos [6], estando determinada en gran medida por la calidad y rapidez de la inteligencia organizacional, y la toma de decisiones [16]. A este respecto la eficacia se configura como una construcción multidimensional [30], debido a que son diversos los aspectos que logran tener un impacto en la eficacia organizativa. Por ende, cabe señalar que esta investigación considera una perspectiva más amplia de la eficacia organizativa, sin limitarse solo a los indicadores económicos, involucrando de esta forma los factores que afectan la calidad de las decisiones.

A este respecto, la eficacia de una decisión estratégica está caracterizada por el proceso de toma de decisiones que se encuentra detrás [3].

\section{Racionalidad y sus determinantes}

La eficacia organizativa se puede medir desde diferentes ópticas, al igual que la calidad de las decisiones. Uno de estos elementos de análisis de la calidad de la decisión es la racionalidad con que se delinea esta, y que puede ser definida como una medida que refleja la intención de que cada elección sea la mejor opción para el logro de los objetivos específicos en una situación determinada [21].

Sin embargo, existen diversas razones por las cuales los seres humanos no toman decisiones racionalmente, ya sea para maximizar la utilidad o el valor. Los métodos racionales de elección en la toma de decisiones se basan sobre un concepto de racionalidad ilimitada, que no logra describir la forma en que las personas naturalmente razonan [20].
Con relación a esto cabe destacar que existe una perspectiva autogenerada o endógena del grado de racionalidad de la toma de decisiones. Bajo este enfoque la racionalidad sería una resultante del propio proceso de interacción entre los equipos de alta dirección.

En este contexto, la flexibilidad cognitiva se configura como el grado en el cual se exploran nuevas ideas o supuestos en el proceso de toma de decisiones. Al mismo tiempo, la flexibilidad cognitiva es considerada como la clave de la innovación [7].

Los resultados de un estudio llevado a cabo por Ying-Fang Su, Shao-Hsi Chung y Shao-Wen Su [38] indican que la flexibilidad cognitiva puede además mitigar la resistencia al cambio organizacional, siendo la visión y la autorreflexión los principales factores que la afectan.

El conflicto cognitivo, por otra parte, puede ser considerado como el catalizador que estimula la creación de nuevo conocimiento en los tomadores de decisiones, lo cual conlleva a un mejor comportamiento organizacional [39]. Asimismo, este existe cuando un grupo de personas discrepa sobre los contenidos de las tareas, incluyendo las diferencias en los puntos de vistas e ideas [18], definiéndose como el grado en el cual existen posiciones o ideas diferentes entre sí en el proceso de toma de decisiones [35]. A este respecto, Jehn, Rispins y Tatcher [17] señalan que la evolución de los diversos conflictos, en los diferentes estados del proceso de toma de decisiones, tiene un impacto sobre el comportamiento del equipo.

No obstante, de acuerdo a los resultados de un estudio realizado por Parayitam y Dooley [26] muestran que el conflicto cognitivo se relaciona con la calidad de la decisión y el compromiso de los equipos en la toma de decisiones estratégicas en una forma curvilínea, es decir, en los niveles bajos y altos de conflicto cognitivo, los equipos son menos productivos que en niveles moderados.

En este sentido, el grado de flexibilidad cognitiva y el grado de conflicto cognitivo se configuran como elementos claves que pueden influir sobre la racionalidad de los procesos de toma de decisiones estratégicas, ya que, tal como lo establece Ramos y Ávila [29], la flexibilidad cognitiva en el proceso 
de toma de decisiones cumple el rol de establecer prioridades, reflexionar y adoptar diferentes estrategias ante una misma situación, con el fin de que este proceso sea capaz de moverse de manera libre e irse adaptando. Es decir, puede entregar más opciones, información y alternativas para la toma de decisión.

En consecuencia, las hipótesis de la investigación son las siguientes:

H-1 La calidad de las decisiones estratégicas se relaciona positivamente con la eficacia de las organizaciones.

La evidencia empírica resultante de las investigaciones de Papadakis Lioukas y Chambers [25], Hiller y Hambrick [14], Arendt, Priem y Ndofor [2] que sustentan la hipótesis. Incluso en países emergentes se ha podido demostrar que existe una relación positiva y significativa entre la calidad de las decisiones y la eficacia organizativa [32].

\section{$H$-2: La racionalidad se relaciona positivamente} con la calidad de las decisiones estratégicas.

Esta relación entre racionalidad y calidad de las decisiones se vincula a que una mayor rigurosidad en la búsqueda y análisis de información permitirá generar y elegir mejores alternativas [8]. Asimismo, los resultados de un estudio con 233 empresas privadas e internacionales de China indican que la racionalidad de la decisión influye de manera positiva en la efectividad de la toma de decisiones [19], y por ende afecta en su calidad.

H-3: La flexibilidad cognitiva y el conflicto cognitivo se relacionan positivamente con el grado de racionalidad del proceso de toma de decisiones estratégicas.

La relación entre flexibilidad cognitiva y racionalidad ha sido poco analizada en la literatura internacional. No obstante, en el último tiempo se ha planteado, aunque no necesariamente se ha corroborado, la idea que mayor apertura cognitiva puede conllevar a una mayor exhaustividad en los procesos decisionales [15].

La vinculación entre conflicto cognitivo y racionalidad no es usual en los estudios internacionales. Si bien hay una serie de investigaciones que proponen a ambas variables como aspectos del proceso de toma de decisiones estratégicas [25, 31], es posible postular que un mayor grado de conflicto de ideas debe conducir a decisiones más exhaustivamente elegidas [34]. Más específicamente, Gunter [12] sugiere que la diversidad de ideas puede conllevar a decisiones más racionales.

Esto es relevante, ya que sin buenos insumos de información, conocimiento, atención, capacidad de análisis, pensamiento profundo, buenas decisiones y resultados difícilmente pueden ser esperados. Los buenos insumos son una condición necesaria, aunque no suficiente, para tomar una buena decisión [5].

La siguiente Figura 1 representa, en forma integrada, el conjunto de hipótesis de la investigación:

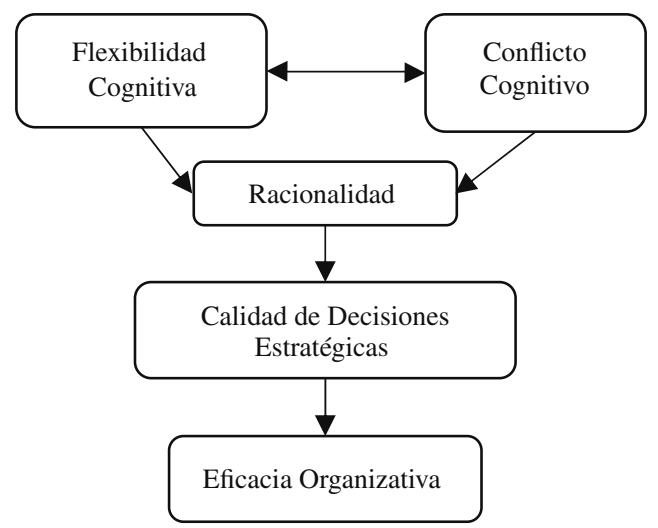

Figura 1. Modelo de la investigación.

Fuente: Elaboración propia.

\section{MÉTODO}

La metodología utilizada es de orden cuantitativo, exploratorio, no representativo y no probabilístico. Como instrumento de recolección de la información se usó un cuestionario, el cual fue dirigido al máximo directivo de la organización, o a un alto directivo que hubiese participado en el proceso de toma de decisiones que fue analizado. El primer contacto con dichas empresas fue a través de una entrevista semiestructurada, lo cual facilitó la aplicación del instrumento. El cuestionario fue aplicado por un grupo de estudiantes de un programa de Magíster en Liderazgo y Gestión de Organizaciones. Los estudiantes insistieron hasta 3 veces en cada empresa (320 en total). Al cabo de 6 meses se consiguieron 96 
respuestas utilizables de empresas de la Región de Arica y Parinacota, que representan una tasa de respuesta del 22,22\%. La unidad de análisis está constituida por la percepción que poseen los directivos sobre el proceso de dirección estratégica de las empresas, siendo el nivel de análisis el ápice estratégico de las empresas pequeñas y medianas de Arica.

Se empleó un conjunto de variables y medidas validadas en investigaciones previas, las cuales se muestran en la Tabla 1, y se usó una escala de tipo Likert (1-7), con el fin de rescatar información relevante de cada empresa. Para cada caso se midió la fiabilidad de las variables y medidas de acuerdo con el cálculo del Alpha de Cronbach, resultando todas en un nivel aceptable de fiabilidad, con valores sobre 0,766 .

Tabla 1. Variables y medidas.

\begin{tabular}{|c|c|}
\hline Variables & Ítems \\
\hline $\begin{array}{l}\text { Eficacia } \\
\text { organizativa } \\
\text { Basado en: } \\
\text { Rodríguez- } \\
\text { Ponce [33] } \\
\text { Alpha de } \\
\text { Cronbach: } \\
0,891\end{array}$ & $\begin{array}{l}\text { La empresa: } \\
\text {-Tiene una sólida posición económica } \\
\text { y financiera. } \\
\text {-La rentabilidad de la empresa es } \\
\text { altamente satisfactoria para los dueños } \\
\text { del capital. } \\
\text {-La imagen y posicionamiento de la } \\
\text { empresa es óptima. } \\
\text {-La empresa satisface en alta medida las } \\
\text { necesidades de sus clientes. }\end{array}$ \\
\hline $\begin{array}{l}\text { Calidad de } \\
\text { la decisión } \\
\text { estratégica } \\
\text { Amason [1] } \\
\text { Alpha de } \\
\text { Cronbach: } \\
0,909\end{array}$ & $\begin{array}{l}\text { La decisión adoptada: } \\
\text {-Fue de máxima calidad. } \\
\text {-Permitió el logro de los objetivos. } \\
\text {-Generó una solución eficaz y eficiente. } \\
\text {-Permitió crear valor y aportar al } \\
\text { desarrollo de la universidad. }\end{array}$ \\
\hline $\begin{array}{l}\text { Racionalidad } \\
\text { Dean y } \\
\text { Sharfman [9] } \\
\text { Alpha de } \\
\text { Cronbach: } \\
0,766\end{array}$ & $\begin{array}{l}\text { En el proceso de toma de decisiones } \\
\text { estratégicas: } \\
\text {-La búsqueda de información ha sido } \\
\text { profunda y exhaustiva. } \\
\text {-El análisis de información ha sido } \\
\text { profundo y exhaustivo. } \\
\text {-La generación y análisis de alternativas } \\
\text { ha sido profunda y exhaustiva. } \\
\text {-La selección de la opción ha sido } \\
\text { rigurosa y basada en un criterio más } \\
\text { analítico que intuitivo. }\end{array}$ \\
\hline $\begin{array}{l}\text { Conflicto } \\
\text { cognitivo } \\
\text { Amason [1] } \\
\text { Alpha de } \\
\text { Cronbach: } \\
0,910\end{array}$ & $\begin{array}{l}\text { La decisión adoptada supuso: } \\
\text {-La discusión de muchas ideas diferentes. } \\
\text {-El análisis de múltiples perspectivas. } \\
\text {-La discusión de enfoques diferentes. } \\
\text {-Muchas diferencias de opinión entre } \\
\text { los participantes. }\end{array}$ \\
\hline
\end{tabular}

\begin{tabular}{|c|c|}
\hline Variables & Ítems \\
\hline $\begin{array}{l}\text { Flexibilidad } \\
\text { Sharfman y } \\
\text { Dean [36] } \\
\text { Alpha de } \\
\text { Cronbach: } \\
0,887\end{array}$ & $\begin{array}{l}\text { En la decisión adoptada: } \\
\text {-Hubo posibilidad de incorporar nueva } \\
\text { información en forma recurrente. } \\
\text {-Las ideas originales fueron bien } \\
\text { acogidas. } \\
\text {-Los directivos que participaron, lo } \\
\text { hicieron en forma amplia y creativa más } \\
\text { allá del ámbito de sus cargos. } \\
\text {-Se reconsideraron las posiciones } \\
\text { iniciales. }\end{array}$ \\
\hline
\end{tabular}

Fuente: Elaboración propia.

Para el análisis se utilizó un modelo de regresión lineal simple según la siguiente ecuación de regresión:

$$
\mathrm{V}_{\mathrm{x}}=\beta_{0}+a \beta_{1}+\varepsilon_{\mathrm{i}}
$$

Donde, $\mathrm{V}_{\mathrm{x}}$ : es la variable dependiente, es decir, la eficacia organizativa; $\beta_{0}$ : constante del modelo; $a$ variable independiente: calidad de la decisión estratégica; $\beta_{1}$ es el factor de ponderación y $\varepsilon_{\mathrm{i}}$ : error residual del modelo.

De modo equivalente, para explicar la calidad del diseño de la decisión estratégica se emplea la siguiente ecuación de regresión:

$$
\mathrm{V}_{\mathrm{x}}=\beta_{0}+b \beta_{1}+\varepsilon_{\mathrm{i}}
$$

Donde, $\mathrm{V}_{\mathrm{x}}$ : es la variable dependiente, es decir, calidad del diseño de la decisión estratégica; $\beta_{0}$ : constante del modelo; $b$ variable independiente: racionalidad del proceso de toma de decisiones estratégicas; $\beta_{1}$ es el factor de ponderación y $\varepsilon_{\mathrm{i}}$ : error residual del modelo.

Asimismo, para explicar la racionalidad del proceso de toma de decisiones estratégicas se aplica un modelo de regresión lineal múltiple, según la siguiente ecuación de regresión.

$$
\mathrm{V}_{\mathrm{x}}=\beta_{0}+c \beta_{1}+d \beta_{2}+\varepsilon_{\mathrm{i}}
$$

Donde, $\mathrm{V}_{\mathrm{x}}$ : es la variable dependiente, es decir, la racionalidad del proceso de toma de decisiones estratégicas; $\beta_{0}$ : constante del modelo; $c$ es la variable independiente denominada conflicto cognitivo; $\beta_{1}$ es su factor de ponderación; $d$ es la variable independiente denominada flexibilidad cognitiva; $\beta_{2}$ es su factor de ponderación; y $\varepsilon_{\mathrm{i}}$ : error residual del modelo. 


\section{RESULTADOS Y DISCUSIÓN}

La Tabla 2 muestra los resultados descriptivos de las variables analizadas. En este sentido destaca que en todos los casos existe una variabilidad en las respuestas significativamente distinta de cero $(\mathrm{p}<0,01)$.

Tabla 2. Análisis descriptivo.

\begin{tabular}{|l|c|c|}
\hline \multicolumn{1}{|c|}{ Variables } & Media & $\begin{array}{c}\text { Desviación } \\
\text { estándar }\end{array}$ \\
\hline Racionalidad & 4,0891 & 1,478 \\
\hline Conflicto cognitivo & 4,010 & 1,699 \\
\hline Flexibilidad & 3,995 & 1,690 \\
\hline $\begin{array}{l}\text { Calidad de la } \\
\text { decisión }\end{array}$ & 4,190 & 1,715 \\
\hline $\begin{array}{l}\text { Eficacia } \\
\text { organizativa }\end{array}$ & 4,302 & 1,650 \\
\hline
\end{tabular}

Fuente: Procesamiento del cuestionario. Elaboración propia.

A continuación la Tabla 3 muestra respectivamente las correlaciones de Pearson entre la calidad de las decisiones estratégicas y la eficacia organizativa, así como los resultados de la aplicación de la ecuación de regresión lineal simple.

Tabla 3. Resumen del modelo de regresión.

\begin{tabular}{|l|c|}
\hline & Eficacia organizativa \\
\hline $\mathrm{R}$ & $0,777^{*}$ \\
\hline $\mathrm{R}$ cuadrado ajustado & 0,599 \\
\hline Test $\mathrm{t}$ constante & 4,139 \\
\hline Coeficiente no estandarizado & 1,170 \\
\hline Coeficiente beta aplicar & 0,000 \\
\hline Test $\mathrm{t}$ & 11,961 \\
\hline
\end{tabular}

Eficacia organizativa $=\beta_{0}+$ Calidad de la decisión $\beta_{1}+\varepsilon_{\mathrm{i}}$.

Fuente: Procesamiento del cuestionario. Elaboración propia.

Variable predictora: (Constante), Calidad de la decisión.

*Significancia al $1 \%$.

Los resultados anteriores muestran que la calidad de las decisiones estratégicas es un determinante estructural de la eficacia organizativa. Su capacidad explicativa llega al 59,9\% ( $\mathrm{p}<0,01)$; es estadísticamente significativo (Test $\mathrm{t}=11,961 ; \mathrm{p}<0,01$ ), aunque lógicamente este modelo simple omite variables (Test $\mathrm{t}$ de la constante $=4,139 ; \mathrm{p}<0,01$ ).
La Tabla 4 muestra las correlaciones de Pearson entre la racionalidad y la calidad de la decisión, así como los resultados de la aplicación de la ecuación de regresión lineal simple.

Tabla 4. Resumen del modelo de regresión.

\begin{tabular}{|l|c|}
\hline & $\begin{array}{c}\text { Calidad de } \\
\text { la decisión }\end{array}$ \\
\hline $\mathrm{R}$ & $0,787^{*}$ \\
\hline $\mathrm{R}$ cuadrado ajustado & 0,615 \\
\hline Test t constante & 1,429 \\
\hline Coeficiente no estandarizado constante & 0,458 \\
\hline $\begin{array}{l}\text { Coeficiente no estandarizado } \\
\text { Racionalidad }\end{array}$ & 0,913 \\
\hline Test t & 12,364 \\
\hline
\end{tabular}

Calidad de la decisión $=\beta_{0}+$ Racionalidad $\beta_{1}+\varepsilon_{\mathrm{i}}$ Fuente: Procesamiento del cuestionario. Elaboración propia.

Variable predictora: (Constante), Racionalidad. $*$ Significancia al $1 \%$.

Los resultados anteriores muestran que la racionalidad del proceso de toma de decisiones estratégicas es un determinante estructural de la calidad de la decisión diseñada. Su capacidad explicativa llega al 61,5\% $(p<0,01)$; $y$ es estadísticamente significativa (Test $t=12,364 ; p<0,01)$. La racionalidad es un factor determinante, a pesar de que no es el único, en términos de la calidad y el éxito de la alternativa elegida. Teniendo esta un efecto positivo en el comportamiento de la organización, pudiendo afectar de esta forma en la calidad de las decisiones.

Este hallazgo es consistente con la literatura dominante sobre el tema $[11,25]$ respecto del impacto de la racionalidad sobre la calidad de las decisiones estratégicas. Sin embargo, la racionalidad en la decisión no es garantía de un buen resultado, sí incrementa sus probabilidades de éxito [5]. Por lo tanto es importante analizar los aspectos que influyen sobre la racionalidad en el proceso de toma de decisiones.

Esto coincide con los planteamientos de Chen y Chien [5] quienes postulan que las personas tratan de tomar decisiones de manera racional, pero que se encuentran limitados por su habilidad de procesar la información.

Las Tablas 5 y 6 muestran respectivamente las relaciones significativas existentes entre la 
racionalidad, el conflicto cognitivo y la flexibilidad cognitiva, así como los resultados de la aplicación de la ecuación de regresión lineal múltiple, donde se visualiza la relación que tienen las dos variables sobre la racionalidad.

Tabla 5. Correlación de Pearson entre racionalidad, conflicto cognitivo y flexibilidad cognitiva.

\begin{tabular}{|l|c|c|c|}
\hline Variables & Racionalidad & $\begin{array}{c}\text { Conflicto } \\
\text { cognitivo }\end{array}$ & $\begin{array}{c}\text { Flexibilidad } \\
\text { cognitiva }\end{array}$ \\
\hline Racionalidad & 1,000 & $0,832^{*}$ & $0,868^{*}$ \\
\hline $\begin{array}{l}\text { Conflicto } \\
\text { cognitivo }\end{array}$ & 0,832 & 1,000 & 0,892 \\
\hline $\begin{array}{l}\text { Flexibilidad } \\
\text { cognitiva }\end{array}$ & 0,868 & 0,892 & 1,000 \\
\hline
\end{tabular}

Fuente: Procesamiento del cuestionario. Elaboración propia; * Significancia al $1 \%$

Tabla 6. Resumen del modelo de regresión.

\begin{tabular}{|l|c|c|c|c|c|}
\hline Concepto & $\begin{array}{c}\text { Coeficiente } \\
\text { no estanda- } \\
\text { rizado }\end{array}$ & $\begin{array}{c}\text { Error } \\
\text { están- } \\
\text { dar }\end{array}$ & Test t & Sig. & $\begin{array}{c}\text { R2 } \\
\text { ajustado }\end{array}$ \\
\hline Constante & 0,951 & 0,193 & 4,920 & 0,000 & \\
\hline $\begin{array}{l}\text { Conflicto } \\
\text { cognitivo }\end{array}$ & 0,246 & 0,096 & 2,563 & 0,012 & \\
\hline $\begin{array}{l}\text { Flexibilidad } \\
\text { cognitiva }\end{array}$ & 0,539 & 0,095 & 5,594 & 0,000 & \\
\hline $\begin{array}{l}\text { Capacidad } \\
\text { Explicativa }\end{array}$ & & & & & $0,764^{*}$ \\
\hline
\end{tabular}

Racionalidad $=\beta_{0}+$ Conflicto cognitivo $\beta_{1}+$ Flexibilidad cognitiva $\beta_{2}+\varepsilon_{\mathrm{i}}$

Fuente: Procesamiento del cuestionario. Elaboración propia.

* Significancia al $1 \%$

Variable dependiente: Racionalidad.

Los resultados anteriores muestran que el conflicto cognitivo (Test $\mathrm{t}=2,563, \mathrm{p}<0,05)$, y principalmente la flexibilidad cognitiva (Test $\mathrm{t}=5,594, \mathrm{p}<0,01$ ), son determinantes estructurales de la racionalidad del proceso de toma de decisiones estratégicas. $\mathrm{Su}$ capacidad explicativa llega al 76,4\% ( $<<0,01)$. Cabe destacar que el modelo omite variables relevantes (Test $\mathrm{t}$ de la constante $=4,920, \mathrm{p}<0,01$ ). De esta forma, el conflicto es el grado en el cual chocan las posiciones cognitivas o afectivas existentes entre los responsables que toman las decisiones [1], las cuales repercuten en la racionalidad de la toma de decisiones.

Por lo tanto, la flexibilidad cognitiva, entendida como el grado con el que los tomadores de decisiones exploran nuevas ideas o supuestos [24], de acuerdo con los resultados identificados, impacta en la racionalidad del proceso de toma de decisiones.

Por consiguiente, la mayoría de las decisiones y acciones del equipo de alta dirección tiene un efecto retardado sobre los determinantes del rendimiento, lo que a su vez tiene un efecto retardado sobre la eficacia de la organización [40].

Los resultados evidencian la importancia de poner atención en los determinantes de la calidad del proceso de toma de decisiones, lo cual coincide con los planteamientos de Chen y Chien [5], quienes señalan que una decisión de buena calidad se refiere a su proceso, es decir, a los pasos de la toma de decisión y no a su resultado real. Por consiguiente, la calidad se puede considerar como la calidad de los pasos de la decisión o procedimientos.

\section{CONCLUSIONES}

La investigación llevada a cabo buscó proporcionar evidencia empírica incremental que contribuye a relaciones estadísticamente significativas en la muestra de estudio. En este sentido, se puede establecer que la calidad de los procesos de toma de decisiones estratégicas se relaciona con la eficacia organizativa. Sin embargo, la calidad de la toma de decisiones se ve determinada por la racionalidad, la cual se relaciona con la calidad de la toma de decisiones.

En efecto, la calidad de las decisiones estratégicas influye sobre la eficacia organizativa, por ende no se refuta la hipótesis de la investigación. Asimismo, se demuestra que la racionalidad del proceso de toma de decisiones estratégicas impacta positivamente sobre la calidad de dichas decisiones.

Por otra parte, un hallazgo propuesto de manera conceptual [36], pero poco comprobado en investigaciones precedentes, hace referencia a la relación positiva y significativa entre la flexibilidad cognitiva y el grado de racionalidad del proceso de toma de decisiones estratégicas. En consecuencia, se puede observar una serie de relaciones que van impactando y repercutiendo en cada uno de los factores de la toma de decisiones, llegando a repercutir finalmente en la eficacia de las organizaciones. 
Por último, es posible indicar que el conflicto de ideas contribuye a lograr mayores niveles de racionalidad en las decisiones estratégicas [34].

Por tanto es posible establecer que para lograr mayores niveles de éxito en la toma de decisiones estratégicas y subsecuentemente mayor eficacia organizativa, los equipos de alta dirección deben favorecer la racionalidad de las decisiones estratégicas, buscando y analizando información para la generación de alternativas. Así como también es recomendable favorecer la flexibilidad cognitiva en el proceso de toma de decisiones estratégicas, fomentando la discusión de muchas ideas diferentes, el análisis de diversas perspectivas y opiniones, junto con favorecer el conflicto cognitivo en el proceso de toma de decisiones estratégicas, es decir, estimulando la discusión de nuevas ideas.

\section{REFERENCIAS}

[1] A. Amason. "Distinguishing the effects of functional and dysfunctional conflict on strategic decision making: Resolving a paradox for top management teams". Academy of Management Journal. Vol. 39, Issue 1, pp. 123-148. 1996.

[2] L. Arendt, R. Priem and H. Ndofor. "A CEO-Adviser Model of Strategic Decision Making”. Journal of Management. Vol. 31, Issue 5, pp. 680-699. 2005.

[3] M. Çetin and D. Pekince. "Perceived procedural rationality and political behaviours in strategic decision making process and organizational commitment triangle". Procedia-Social and Behavioral Sciences. Vol. 24, pp. 1154-1163. 2011.

[4] T. Chang and T. Wang. "Using the fuzzy multi-criteria decision making approach for measuring the possibility of successful knowledge management". Information Sciences. Vol. 179, Issue 4, pp. 355-370. 2009.

[5] P. Chen and C. Chien. "What Constitutes “A Quality Decision?”. Journal of Quality. Vol. 16, Issue 2, pp. 87-94. 2009.

[6] R. Daft. "Organizational theory and design". West Publishing. St. Paul, Minnesota, USA. 1995.

[7] E. Dane. "Reconsidering the trade-off between expertise and flexibility: A cognitive entrenchment perspective". Academy of
Management Review. Vol. 35, Issue 4, pp. 579-603. 2010.

[8] H. De Boer, J. Enders and L. Leisyte. "Public sector reform in Dutch higher education: the organizational transformation of the university". Public Administration. Vol. 85, Issue 1, pp. 27-46. 2007.

[9] J. Dean and M. Sharfman. "Procedural Rationality in strategic decision-making process". Journal of Management Studies. Vol. 30, Issue 4, pp. 587-610. 1993.

[10] L. Franz and M. Kramer. "The Dimensions of Decisions: A Conceptual and Empirical Investigation”. In P. Nutt and D. Wilson. Handbook of decision making. Wiley United Kingdompp, pp. 517-540. 2010.

[11] I. Goll and A. Rasheed. "The relationships between top management demographic characteristics, rational decision making, environmental munificence, and firm performance". Organization Studies. Vol. 26, Issue 7, pp. 999-1023. 2005.

[12] H. Gunter. "Educational Leadership and the Challenge of Diversity". Educational Management Administration \& Leadership. Vol. 34, Issue 2, pp. 257-268. 2006.

[13] D. Hambrick and P. Mason. "Upper echelons theory: an update". The Academy of Management Review. Vol. 32, Issue 2, pp. 334-343. 2007.

[14] N. Hiller and D. Hambrick. "Conceptualizing executive hubris: the role of (hyper-) core self-evaluations in strategic decision-making". Strategic Management Journal. Vol. 26, Issue 4, pp. 297-319. 2005.

[15] R. Horan. "The Relationship between Creativity and Intelligence: A Combined Yogic-Scientific Approach". Creativity Research Journal. Vol. 19, Issue 2-3, pp. 179202. 2007.

[16] G. Huber. "Information Technology and Organizational Decision Making”. In R. Galliers and D. Leidner (Ed) Strategic Information Management, p. 640. Routeladge, Oxford: UK. 2003.

[17] K. Jehn, S. Rispins and S. Thatcher. "The effects of conflict asymmetry on workgroup and individual outcomes". Academy of Management Journal. Vol. 53, Issue 3, pp. 596-616. 2010.

[18] K. Jehn. "A multimethod examination of the benefits and detriments of intragroup 
conflict". Administrative Science Quaterly. Vol. 40, Issue 2, pp. 530-557. 1995.

[19] J. Ji and P. Dimitratos. "An empirical investigation into international entry mode decision-making effectiveness". International Business Review. Vol. 22, Issue 6, pp. 9941007. 2013.

[20] D. Jonassen. "Designing for decision making". Educational Technology Research and Development. Vol. 60, Issue 2, pp. 341359. 2012.

[21] R. Mitchell, D. Shepherd and M. Sharfman. "Erratic strategic decisions: when and why managers are inconsistent in strategic decision making". Strategic Management Journal. Vol. 32, Issue 7, pp. 683-704. 2011.

[22] P. Nutt. "Making decision-making research matter: Some issues and remedies". Management Research Review. Vol. 34, Issue 1, pp. 5-16. 2011.

[23] P. Nutt. "Investigating the Success of Decision Making Processes". Journal of Management Studies. Vol. 45, Issue 2, pp. 425-455. 2008.

[24] P. Nutt. "Why Decisions Fail: Avoiding the Blunders and Traps That Lead to Debacles". San Francisco California: Berret-Koehler Publisher, p. 350. 2002.

[25] V. Papadakis, S. Lioukas and D. Chambers. "Strategic decision-making processes: the role of management and context". Strategic Management Journal. Vol. 19, Issue 2, pp. 115-147. 1998.

[26] S. Parayitam and R. Dooley.” Is too much cognitive conflict in strategic decision-making teams too bad?". International Journal of Conflict Management. Vol. 22, Issue 4, pp. 342-357. 2011.

[27] L. Pedraja-Rejas y E. Rodríguez-Ponce. "Estudio comparativo de la influencia del estilo de liderazgo y la congruencia de valores en la eficacia de empresas privadas e instituciones públicas”. Interciencia. Vol. 33 $\mathrm{N}^{\mathrm{o}}$ 1, pp. 8-13. Enero 2008.

[28] L. Pedraja-Rejas y E. RodríguezPonce. "Estilos de liderazgo, gestión del conocimiento y diseño de la estrategia: un estudio empírico en pequeñas y medianas empresas". Interciencia. Vol. $33 \mathrm{~N}^{\circ}$ 9, pp. 651657. Septiembre 2008.

[29] K. Ramos and E. Ávila. "Higher education in management: reinventing the paradigm to gain the capacity to handle today's complexity". On the Horizon. Vol. 18, Issue 1, pp. 45-52. 2010.

[30] P. Richard, T. Devinney, G. Yip and G. Johnson. "Measuring Organizational Performance: Towards Methodological Best Practice". Journal of Management. Vol. 35, Issue 3, pp. 718-804. 2009.

[31] E. Rodríguez-Ponce and L. Pedraja-Rejas. "Quality of strategic decision making: An empirical study of behavioral variables". African Journal of Business Management. Vol. 6, Issue 34, pp. 9704-9717. 2012.

[32] E. Rodríguez-Ponce y L. Pedraja-Rejas. "Efectos e implicaciones de las decisiones estratégicas en las instituciones universitarias". Interciencia. Vol. $32 \mathrm{~N}^{\circ}$ 9, pp. 593-600. 2007.

[33] E. Rodríguez-Ponce. "Gestión del conocimiento y eficacia de las organizaciones: Un estudio empírico en instituciones públicas". Interciencia. Vol. $32 \mathrm{~N}^{\circ} 12$, pp. 820-826. 2007

[34] E. Rodríguez-Ponce. "El proceso de la toma de decisiones estratégicas en las universidades públicas". Revista Calidad en la Educación. No 24, pp. 49-63. 2006.

[35] E. Rodríguez-Ponce. "La toma de decisiones estratégicas: una perspectiva integradora". Ediciones Consorcio de Universidades Estatales. Santiago, Chile. 2004.

[36] M. Sharfman and J. Dean. "Flexibility in strategic decision making". Journal of Management Studies. Vol. 34, Issue 2, pp. 191-217. 1997.

[37] R. Slotegraaf and K. Atuahene-Gima. "Product development team stability and new product advantage: The role of decision-making processes". Journal of Marketing. Vol. 75, Issue 1, pp. 96-108. 2011.

[38] Y.-F Su, S.-H. Chung and S.-W. Su. "The impact of cognitive flexibility on resistance to organizational change". Social Behavior and Personality. Vol. 40, Issue 5, pp. 735745. 2012.

[39] J. Woods. "Using cognitive conflict to promote the use of dialectical learning for strategic decision-makers". The Learning Organization. Vol. 19, Issue 2, pp. 134-147. 2012.

[40] G. Yukl. "How leaders influence organizational effectiveness". The Leadership Quarterly. Vol. 19, pp. 708-722. 2008. 